\title{
Editorial: Apoptosis Is Critical to Pain Control
}

\author{
James David Adams \\ School of Pharmacy, University of Southern California, Los Angeles, USA \\ Email: jadams@usc.edu
}

Received May 17, 2013; revised June 17, 2013; accepted June 24, 2013

Copyright (c) 2013 James David Adams. This is an open access article distributed under the Creative Commons Attribution License, which permits unrestricted use, distribution, and reproduction in any medium, provided the original work is properly cited.

\section{Introduction}

Dear Readers-Pain frequently involves a neural circuit that can either magnify or suppress pain, depending on the way the brain processes the pain signals and depending on the pain causing or pain suppressing compounds that are secreted in the skin. Pain is essential to life, since it helps us to avoid damaging situations. The most abundant pain sensors are in the skin, and are called the transient receptor potential cation channels (TRP). The TRP family contains many members such as the vanilloid receptors (TRPV), the canonical receptors (TRPC), the ankyrin repeat receptors (TRPA), the polycystin recaptors (TRPP), the melastatin receptors (TRPM) and the mucolipin receptors (TRPML) [1]. These cation channels allow cations to cross the plasma membrane, such as sodium and calcium. In the skin, these receptors are found on small unmyelinated (C-type) or thinly myelinated (A delta-type) nerve terminals [2]. Their cell bodies are in the dorsal root ganglion with dendrites that form synapses in the spinal cord. These synapses are formed with ascending CNS neurons that have cell bodies in the hypothalamus, hippocampus and other areas. These CNS neurons form synapses with other CNS descending neurons that form synapses in the spinal cord with descending neurons that have nerve terminals in the skin [2]. These descending neurons are neurotrophin secreting neurons.

TRP channels are activated by histamine, bradykinin, fatty acid metabolites, endocannabinoids, cannabinoids, ATP, acid, heat, cold, prostaglandins, cytokines, capsaicin, monoterpenoids and other stimuli. The initial sensation can be heat, cold, pain or itching that can be several minutes in duration. Usually, prolonged activation of the TRP channels, for more than 20 - 30 minutes or so, results in long term deactivation and pain relief. This is how capsaicin and the monoterpenoids, such as menthol, relieve pain [2]. Initial receptor activation is followed by long term deactivation. Arachidonic acid produces pain causing prostaglandins and pain relieving resolvins, in a balance. Resolvins deactivate TRP channels resulting in pain relief [3]. Endocannabinoids, prostaglandins, resolvins, histamine, bradykinin, cytokines, ATP and other endogenous compounds are produced where they are needed and have short durations of action. This natural pain controlling system can be altered by nonsteroidal anti-inflammatory drugs (NSAIDS) and other drugs, resulting in the inability to control pain naturally. This can be disastrous for patients.

Pain can be blocked at any point in the neural circuit. Opioids block pain in the brain. NSAIDS block pain in the skin and other sites. Menthol and other monoterpenoids block pain in the skin, where they are applied. Monoterpenoids are probably safer than opioids and NSAIDS since monoterpenoids are applied in small amounts to the skin. Opioids and NSAIDS are taken orally in large doses and penetrate into many organs of the body. As of 2013, prescription opioids kill 14,000 patients yearly. As of 2009, NSAIDS cause 100,000 ulcers and 10,000 deaths annually.

TRP channels allow calcium to penetrate into nerve terminals. This can activate apoptosis mechanisms that result in nerve terminal destruction [4]. This nerve terminal destruction in the skin is a prominent feature of Adams disease, a disease that involves severe pain from over activation of TRP channels by cold and other stimuli [5]. Adams disease appears to be caused by dysfunctional TRP receptors in the skin. Destruction of nerve terminals in the skin may cause long term pain relief.

The descending neurons that terminate in the skin secrete neurotrophins that are essential for the regrowth of TRP containing nerve terminals. The neurotrophin secreting nerve terminals help reestablish the neural circuit involved in pain sensation.

\section{REFERENCES}

[1] M. Moran, M. McAlexander, T. Biro and A. Szallasi, "Transient Receptor Potential Channels as Therapeutic Targets," Nature Reviews in Drug Discovery, Vol. 10, No. 8, 2011, pp. 601-620. doi:10.1038/nrd3456

[2] M. Schumacher, “Transient Receptor Potential Channels 
in Pain and Inflammation: Therapeutic Opportunities," Pain Practice, Vol. 10, No. 3, 2010, pp. 185-200. doi:10.1111/j.1533-2500.2010.00358.x

[3] S. Bang, S. Yoo, T. Yang, H. Cho, Y. Kim and S. Hwang, "Resolvin D1 Attenuates Activation of Sensory Transient Receptor Potential Channels Leading to Multiple Antinociception," British Journal of Pharmacology, Vol. 161, No. 3, 2010, pp. 707-720. doi:10.1111/j.1476-5381.2010.00909.x
[4] G. Shapovalov, V. Lehen'kyi, R. Skryma and N. Prevarskaya, "TRP Channels in Cell Survival and Cell Death in Normal and Transformed Cells," Cell Calcium, Vol. 50, No. 3, 2011, pp. 295-302. doi:10.1016/j.ceca.2011.05.006

[5] J. Adams, “Adams Disease: Description and Management with Capsaicin and Heat," The Open Complementary Medicine Journal, Vol. 3, 2011, pp. 10-12. doi:10.2174/1876391X01103010010 